\title{
Correction: The Smart Life Stay (SLS) program: effects of a lifestyle intervention program in combination with health tourism and health guidance for type 2 diabetes
}

Madoka Matsushita, Akiko Muramoto, Eri Nomura, Yukari Eguchi, Ayako Kato, Yoshiko Sano, Mai Kabayama, Masashi Arakawa, Yuko Oguma, Daisuke Yabe, Masaaki Matsunaga, Hiroshi Yatsuya, Hiroshi Arima and Kazuyo Tsushita

Correction to: Nutrition \& Diabetes https://doi.org/10.1038/s41387-020-00136-x published online 29 August 2020

Following publication, the authors asked to add the following institution to the affiliations of author Madoka Matsushita:
Department of Endocrinology and Diabetes, Nagoya University Graduate School of Medicine

Both the PDF and HTML versions of the Article have been updated accordingly.

Published online: 23 September 2020 